\section{TGLㄴ}

Recibido:

Octubre 2013

Publicado:

Enero 2014

ISSN: $2340-8685$

$\mathrm{N}^{\circ}$ DEPÓSITO LEGAL:

MA 1949-2013

Pág 33 a la 48

Palabras clave

fluidez lectora, velocidad lectora, evaluación formativa, rúbrica, coevaluación

\section{Keywords}

reading fluency, reading rate, formative assessment, rubric, peer assessment.

\title{
Fluidez Lectora y Evaluación Formativa
}

\author{
Andrés Calero
}

Psicopedagogo

\section{Resumen}

Existe un consenso generalizado entre los docentes, en el sentido de que la lectura fluida es una de las destrezas más importantes a desarrollar en los primeros años de escolaridad. Sin embargo, no existe una definición clara del constructo fluidez lectora en el ámbito de la investigación y en el medio escolar que oriente la práctica en este aprendizaje y su evaluación. Mientras algunos especialistas apoyan una definición simple de la fluidez lectora basada en la velocidad lectora, otros plantean que la lectura prosódica, relacionándola con la lectura expresiva. En este artículo reflexionamos acerca del hecho de que una lectura veloz no siempre es sinónimo de lectura fluida, y que el énfasis que tradicionalmente se ha puesto en la velocidad al leer en las escuelas -un aspecto singular de la lectura fluida-, ha llevado a muchos docentes y alumnos a pensar que leer con fluidez es leer deprisa.

Valoramos también la importancia de una evaluación formativa que promueva la coevaluación y la autoevaluación, para que los lectores obtengan un conocimiento exacto de cuáles son las demandas que se le exigen para llevar a cabo una lectura fluida, y sean capaces de controlar su propio aprendizaje. Finalmente, abogamos por el uso de la rúbrica, como un instrumento de evaluación cualitativa y formativa, para guiar el proceso de evaluación de la fluidez lectora.

\section{Summary}

Despite there is a general agreement that reading fluency is one the most important skills to be considered in the development of early literacy adquisition, the construct of fluency has been criticized for lacking a clear definition that informs the learning process among reading scholars and teachers. Whereas some researchers is advocates of a simple definition of reading fluency that centers on rate as the main ability to be mastered by students, others argue that prosodic or expressive oral reading is a skill not to be overlooked by teachers in classrooms.

In this article, we reflect on the fact that fast reading is not always fluent reading and that the traditional overemphasis on teaching reading rate in schools -just one aspect of reading fluency-, has led many teachers and students to think of reading fluency as simply reading speed. We then consider formative assessment to promote peer and self assessment, in order for the readers to be able to gain a clear understanding of the standards for good reading fluency perfomance, and to monitor their own perfomance. Finally, we propose the use of quality rubrics to guide assessment. 


\section{Introducción}

Aunque la mayoría de docentes de Educación Primaria pueden distinguir entre una lectura oral fluida y otra que no lo es, la naturaleza específica del constructo fluidez lectora sigue siendo un misterio para muchos de ellos. Casi un siglo de influencia en la escuela de un enfoque de aprendizaje que ha puesto un énfasis especial en el desarrollo de habilidades lectoras, ha implantado en el imaginario colectivo de profesores y alumnos de Educación Primaria determinadas prácticas de enseñanza y evaluación, en línea con la idea de que una lectura fluida es equiparable a una lectura veloz, que leer es básicamente leer deprisa o, lo que es lo mismo, tener una buena velocidad lectora asociada a un cronómetro. De otro modo, se ha establecido una fácil y cuestionable equivalencia entre fluidez lectora y velocidad lectora. Sin embargo, la velocidad lectora no es sino un aspecto, un indicador singular de la fluidez lectora. La lectura fluida es algo más, es el reconocimiento armonioso y expresivo de las palabras del texto que leemos, para comprenderlo, incompatible muchas veces con la velocidad lectora que los cronómetros en las aulas miden. En otras palabras, la velocidad lectora es una condición necesaria, pero no suficiente, de medida de una adecuada fluidez lectora.

Es, por tanto, ineludible un debate profesional a fondo, que renueve el currículo del aprendizaje de la lectura, para situar el concepto de la fluidez lectora y el desarrollo de procedimientos de evaluación de esta destreza, en contextos auténticos y significativos de lectura, que refuercen y mejoren en los alumnos el aprendizaje del automatismo en el reconocimiento de palabras, la lectura prosódica, y la comprensión del texto. Una evaluación formativa que, además, implique a los estudiantes en el manejo de estrategias de autoevaluación, con el objetivo de que adquieran el suficiente conocimiento metacognitivo que les ayude a controlar y autorregular su propio progreso en ese aprendizaje.

En consecuencia, en esta colaboración con Investigaciones sobre Lectura contribuimos a ese debate, planteándonos dos objetivos: a) Reflexionar sobre el concepto de fluidez lectora a la luz de los datos de la investigación actual, y b) Sugerir procedimientos de evaluación formativa, que promuevan el desarrollo metacognitivo de los alumnos de Educación Primaria, a través de instrumentos que induzcan en ellos el uso de procesos de pensamiento estratégicos, orientados a la mejora y la autorregulación de su competencia en lectura fluida y la comprensión del texto.

\section{La fluidez lectora y sus componentes}

A comienzos de este siglo XXI, la Comisión Nacional de Lectura (NRP), creada a petición del Congreso de EEUU en el año 2000, e integrada por 14 expertos en el ámbito del aprendizaje la lectura, acumuló suficientes evidencias experimentales para concluir que la fluidez lectora representa un aspecto esencial de la competencia lectora de los estudiantes, junto con otros más como la conciencia fonémica, el vocabulario, la decodificación, el reconocimiento de palabras y la comprensión lectora. Entre sus conclusiones, se recomendaba explícitamente que se promovieran diseños pedagógicos de enseñanzaaprendizaje y evaluación de la fluidez lectora, basados en los datos que la investigación aporta. Desde el Informe de esa Comisión hasta hoy, son muchos los trabajos de investigación que han concluido la importancia de un aprendizaje temprano de la fluidez lectora, y el uso de instrumentos de evaluación de carácter formativo. 
Señalar inicialmente que el Diccionario de Lectura de Harris \& Hodges, (1995:510) define la lectura fluida como:"una habilidad eficiente en el reconocimiento de palabras, que permite al lector construir el significado del texto. La fluidez se manifiesta como una lectura oral segura, rápida y expresiva".

Desde el punto de vista del desarrollo individual de esta competencia, esa habilidad puede ser descrita, de acuerdo con Rasinski et al. (2011a ; 2011b), con la metáfora del tránsito que sigue un lector a lo largo de un puente, desde las primeras etapas de aprendizaje de la decodificación de palabras, hasta que finalmente alcanza la suficiente competencia en comprensión de textos (figura 1). De ese modo, en el ámbito de la investigación actual (Kuhn \& Stahl, 2003; Pikulski y Chard, 2005; Miller \& Schwanenflugel, 2006) se conviene que, una vez que los estudiantes han aprendido el código alfabético, la fluidez lectora la adquieren a través de la mejora de su competencia en dos destreza lectoras estrechamente asociados al logro de un adecuado nivel de comprensión lectora:

a) El automatismo en el reconocimiento de palabras.

b) La prosodia o expresividad en la lectura del texto.

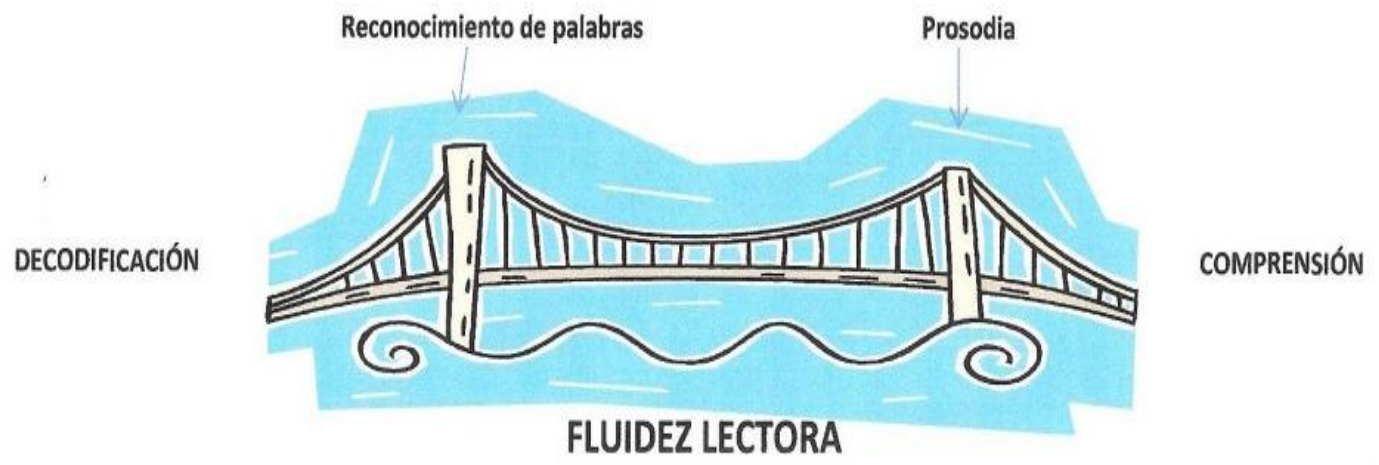

Figura 1: Puente entre la decodificación y la comprensión.

Podríamos caracterizar la lectura fluida, como aquella que se hace sin errores en el reconocimiento y decodificación de palabras, con un adecuado ritmo y expresión, acelerando o deteniendo la lectura cuando convenga para buscar el sentido del texto, un fraseo adecuado haciendo las pausas pertinentes en función de las marcas gráficas, y diferenciando el tono de la lectura de determinadas palabras o frases para apoyar la comprensión del texto. Es decir, además de una buena capacidad de reconocimiento automático de palabras (velocidad lectora), los lectores fluidos suelen aportar a la tarea un uso activo e intencionado de su capacidad para frasear y usar el tono y la expresión adecuada a las palabras o frases del texto, que les ayuden a extraer el significado del mismo (prosodia). En consecuencia, la lectura fluida no puede convertirse en una simple y monótona actividad de decodificación y reconocimiento de palabras, sin aporte de pensamiento estratégico alguno sobre lo que el estudiante está leyendo, y lo que es más importante, sobre cómo está leyendo. Este es el atributo diferencial de una lectura fluida, el secreto, el punto en el que la fluidez lectora conecta directamente con la comprensión a través del componente prosódico, como la mejor herramienta estratégica para que el estudiante ponga el foco de atención en el significado a través del fraseo y la expresión adecuada del texto que lee.

El automatismo es ese aspecto de la fluidez lectora que caracteriza a un lector que no sólo lee las palabras con precisión y exactitud, sino también sin esfuerzo alguno, con rapidez. 
Una buena competencia en esta destreza libera los recursos cognitivos del alumno para dedicarlos a la tarea importante del seguimiento y control de la comprensión del texto (LaBerge \& Samuels (1974) ¿Cómo se logra este automatismo? Sin duda alguna a través del encuentro frecuente con las palabras escritas, mediante procedimientos de lecturas repetidas en la escuela y en la familia (Calero, 2013a). Cada uno de estos encuentros genera una representación mental de dichas palabras en la memoria de trabajo del lector. Una vez que se incrementa el encuentro con esas palabras, se automatiza progresivamente el reconocimiento de dichas representaciones mentales, para así poder recuperarlas durante la lectura.

El automatismo en fluidez lectora se puede determinar a través de la medida de la velocidad con la que los alumnos leen un texto. Todos estaríamos de acuerdo en que la mejora de la comprensión lectora en las etapas iniciales del aprendizaje de la lectura está fuertemente determinada por el automatismo. Así, los lectores retrasados leen menos palabras por unidad de tiempo, que aquellos otros cuya lectura mantiene un ritmo adecuado. Como consecuencia, los alumnos más lentos invierten más tiempo y energías en la tarea de recuperar de la memoria aquellas palabras del texto que leen, y tienen más dificultades para hacer un seguimiento y control de la comprensión de aquellas ideas que el propio texto les va sugiriendo. Esa situación lleva a estos estudiantes a leer cada vez menos, y a generarles un retraso progresivo durante su escolaridad con respecto a otros compañeros de aula que progresan adecuadamente en este aprendizaje.

Por otra parte, es un hecho que las evidencias experimentales acumuladas durante el pasado siglo, en relación a la existencia de una correlación positiva entre velocidad de palabras leídas por minuto y comprensión lectora, han condicionado tradicionalmente en la escuela el diseño y la elaboración de programas de enseñanza y evaluación de la fluidez lectora basados en el desarrollo y la medida de la velocidad lectora, olvidándose otras variables como la prosodia y la comprensión (González Trujillo, 2005). Sin embargo, también es un hecho que la existencia de una correlación entre dos variables de aprendizaje no implica una relación causa-efecto entre ellas. La velocidad lectora no es sino un aspecto, un indicador singular de la fluidez lectora. Un lector puede tener una lectura poco fluida leyendo a una velocidad alta, dado que no focaliza sus energías cognitivas en el manejo de las destrezas prosódicas (entonación, fraseo adecuado acento, pausas, etc.), viéndose afectada su capacidad de comprensión del texto. Rasinski (2011a: 96) establece una comparación entre la lectura que realiza este tipo de lector, con la conducción de un vehículo en punto muerto: "El conductor puede poner el motor del vehículo girando a miles de revoluciones por minuto, pero hasta que el cambio de marchas no se active, el vehículo no se moverá. Estos lectores no suelen engranar, o ensamblar la comprensión a la lectura que hacen. Algunos estudiantes poseen una comprensión pobre y un adecuado nivel de velocidad lectora porque, como en el caso del conductor que no pone el cambio de marchas, no han conectado su capacidad de decodificación con la comprensión del texto".

La prosodia es ese ámbito de la fonología que singulariza aquellos rasgos particulares de determinados segmentos del lenguaje oral y escrito, y que se manifiesta a través del acento silábico, la entonación mayor o menor de palabras o frases, y la duración en la pronunciación de las mismas. El componente prosódico de la fluidez lectora es ese elemento que echa en falta Rasinski, que caracteriza a un lector que lee asemejando su lectura al lenguaje oral, con la suficiente expresividad, fraseo y entonación, para conectar el automatismo en el reconocimiento de palabras con la comprensión del texto. La prosodia ayuda a los lectores a comprender el texto de varios modos: por ejemplo, cuando enfatizan determinadas palabras, 
cuando frasean deslindando claramente en su lectura determinados segmentos del texto, o cuando marcan su carácter interrogativo o exclamativo.

Dos evidencias abonan la idea del peso específico del componentes prosódico para el niño en el aprendizaje de la fluidez lectora. Por un lado, la experiencia nos muestra a los docentes que cuando un alumno lee prosódicamente respetando las pausas, las marcas textuales de expresividad, o el fraseo correcto del texto, etc., acostumbra a poner en juego destrezas metacognitivas de supervisión y control de la comprensión de lo que está leyendo. Es decir, la lectura prosódica es indicativa de la habilidad del niño para controlar el sentido del texto, segmentándolo de un modo intencionado en función de sus elementos sintácticos (fraseo adecuado), y semánticos (entonación de determinadas palabras o frases, etc). Por otra parte, es curioso observar cómo, desde el nacimiento, los niños son especialmente sensibles a los rasgos prosódicos del lenguaje oral que están aprendiendo. Cuando el padre o la madre acentúan expresivamente una palabra en una frase ("mira... iiPERRO!!), los bebés son especialmente receptivos a esa palabra para construir su significado, usando esos rasgos prosódicos como auténticas pistas de conocimiento fonético en la estructura sintáctica del lenguaje oral que perciben. Numerosos estudios han demostrado esa capacidad de los niños pequeños (Hirsh et al., 1987; Christophe et al., 2001). Dadas esas evidencias, que demuestran que los niños construyen su lenguaje oral a partir de su sensibilidad a los rasgos prosódicos del habla de los demás, uno se plantea dos interrogantes: ¿Qué ocurre cuando los prelectores descubren que lo escrito también es lenguaje oral, que también contiene significado? ¿Anestesiamos en la escuela esa sensibilidad? ¿La aprovechamos los maestros, para utilizarla también durante el aprendizaje de la lectura en los primeros años de Educación Primaria, y así favorecer el reconocimiento de palabras y, en definitiva, el desarrollo de la fluidez lectora? Nunca, como en esos momentos, el niño podría implicarse cognitivamente mejor en el aprendizaje del lenguaje escrito, con el bagaje de conocimiento prosódico del lenguaje oral que trae a la escuela. La realidad es que en bastantes aulas no se incorpora esa destreza al proceso de enseñanza de la fluidez lectora en esa fase inicial, reduciéndolo a la mera adquisición de un buen nivel de velocidad lectora para, supuestamente, posibilitar así la comprensión ulterior del texto.

Diversas investigaciones resaltan la incidencia de la enseñanza de lectura prosódica en el logro de la comprensión. Rasinski, (1985), encontró una correlación alta entre los resultados de lectura prosódica medidos a través de una rúbrica y una prueba estandarizada de comprensión lectora, en un trabajo llevado a cabo con alumnos de $3^{\circ}$ y $5^{\circ}$ de Educación Primaria, $(\mathrm{r}=.74$ y $\mathrm{r}=.73$ respectivamente). Otros trabajos, como los de Miller \& Schwanenflugel, op.cit.; Schwanenflugel et al., 2004; González Trujillo, op.cit.; Ravid \& Mashraki, 2007), concluyen que los buenos lectores tienen en cuenta los aspectos prosódicos de la lectura, muestran una entonación y expresividad adecuada con los distintos segmentos del texto, y además presentan niveles superiores de comprensión lectora. En otras palabras, se comprueba que una vez que el niño ha conseguido un cierto nivel de automatismo en el reconocimiento de palabras (velocidad lectora), la lectura prosódica es un componente de la fluidez que explica una varianza adicional importante en el logro de la comprensión lectora. Básicamente, porque la lectura fluida comporta no sólo saber cuándo aumentar el ritmo lector, o disminuirlo para buscar el sentido a lo que se está leyendo; sino que además conlleva un uso consciente de la puntuación o las marcas expresivas del texto, y saber dónde poner énfasis al leer para resaltar el significado de determinadas palabras o frases, situando así el automatismo y la lectura prosódica al servicio de la construcción de comprensión lectora. 


\section{Evaluación de la fluidez lectora: Objetivos y características}

En Educación Primaria, la evaluación debería apoyar la consecución del aprendizaje en lugar de simplemente medirlo. Este principio básico de la evaluación formativa suele olvidarse con frecuencia, sobre todo cuando se trata de estimar el logro del estudiante en nivel lector. Entre otras destrezas esenciales del aprendizaje de la lectura (reconocimiento de palabras, fluidez lectora, vocabulario y comprensión lectora), la fluidez lectora ha sido tradicionalmente una de las que más se ha evaluado, y menos se ha enseñado en las escuelas.

Sin embargo, esa evaluación se ha basado en el rendimiento del estudiante en precisión y velocidad lectora en 1 minuto. Así, el procedimiento prevalente de evaluación de la fluidez lectora en la escuela ha situado al profesor (con su cronómetro), como el único responsable del seguimiento, control y evaluación de un aspecto singular de este aprendizaje: la velocidad lectora. Los análisis estadísticos de resultados en velocidad lectora, han estipulado que aquellos alumnos que obtienen resultados normativos ajustados o por encima del percentil 50, son considerados como lectores con un nivel de automatismo adecuado al curso en que están. Como consecuencia, por muchas escuelas circulan tablas que contienen el número de palabras por minuto que los alumnos de los distintos niveles tienen que conseguir, para ser considerados como lectores fluidos. De ese modo, con este enfoque de la evaluación de la fluidez lectora se sitúa al docente como el único responsable de emitir juicios acerca del nivel de fluidez lectora del alumno. Éste, se limita a "correr", y en muchos casos a "ladrar el texto", leyendo generalmente para satisfacer las expectativas y objetivos de lectura de su "profe", o de su familia. En esa situación de evaluación, el único mensaje que el docente transmite, y que el alumno suele aprender, es que leer velozmente equivale a tener un buen rendimiento lector. Algunas de las interrogantes a plantear ante ese procedimiento erróneo de evaluar la fluidez lectora son:

- ¿Es ese un modelo de evaluación válido, fiable y eficiente para que los niños mejoren su fluidez y comprensión lectora?

- ¿Cómo docentes, qué decisiones pedagógicas significativas podemos adoptar con aquellos alumnos que "no leen deprisa", a partir de los resultados que obtenemos del uso del cronómetro?

- ¿Puede considerarse siempre la lectura lenta y reposada como un problema de aprendizaje, o de retraso lector?

- ¿Cuándo, y con qué criterios válidos y fiables se decide que un alumno es lento al leer?

- ¿Qué sentido, o efecto tiene construir y divulgar tablas o datos normativos que sitúen el número de palabras por minuto que un alumno tipo debe leer en un nivel educativo concreto, si no es el de motivar la generalización de prácticas escolares de aprendizaje de la fluidez lectora no fundamentadas pedagógicamente en la investigación?

- ¿Tiene ese modelo de evaluación algún significado para el logro del desarrollo cognitivo o metacognitivo del lector inicial?

La consecuencia más importante de ese enfoque de la evaluación es que, en el tránsito del aprendizaje que realizan los lectores iniciales desde el reconocimiento de palabras y la fluidez lectora hasta llegar a la comprensión del texto, aproximadamente un $25 \%$ de ellos no alcanzan a desarrollar una lectura fluida que les prepare para entender lo que leen. Una explicación posible de ese tránsito fallido está en el hecho de que, en los cursos iniciales de Educación Primaria, no se ofrecen suficientes posibilidades a los lectores para aprender 
estrategias de fluidez lectora (dimensión cognitiva); y menos aún se les brindan oportunidades para que también aprendan el sentido que tiene una lectura fluida y cómo mejorarla (dimensión metacognitiva), a través de procedimientos de evaluación formativa (Calero, 2012).

Esas prácticas de evaluación de la fluidez lectora suelen carecer de significatividad para muchos lectores, y para el propio proceso de enseñanza-aprendizaje dado que, por un lado, muchos estudiantes acostumbran a focalizar su atención casi con exclusividad en la gestión de su velocidad lectora, y no en la comprensión del texto; y por otro, no proporcionan retroalimentación de carácter formativo ni al docente ni al alumno, para la oportuna toma de decisiones de ambos en favor de la mejora en este aprendizaje. Ocurre, además, que muchos lectores habituados a ellas las consideran monótonas, aburridas y carentes de interés. De igual modo, bastantes docentes terminan también generando la creencia equivocada de que la velocidad lectora es la causa de una buena fluidez lectora, que en la velocidad lectora está el germen de la fluidez lectora y la comprensión. Una incidencia negativa en este estado de creencias la han tenido, en general, determinados programas de desarrollo de la fluidez lectora que se han "comercializado" en bastantes escuelas. En muchos de estos programas, se parte de la idea errónea que considera el incremento en velocidad lectora como la causa, y no como la consecuencia de una lectura fluida; es decir, se asume que si la velocidad se incrementa, la fluidez mejorará también. No obstante, "las auténticas consecuencias" de ese error de perspectiva pedagógica son dramáticas para bastantes estudiantes de Educación Primaria (del Mazo (2013). Exigir a los alumnos que lean con velocidad supone, en muchos casos, que ambas: su lectura prosódica y su comprensión lectora sufran, se vean disminuidas de un modo significativo.

Por el contrario, la propuesta de evaluación formativa que aquí proponemos se plantea desde la idea de:

- Una evaluación que forme parte del proceso de aprendizaje de la fluidez lectora.

- El lector como un participante activo en procedimientos de evaluación para aprender.

- La utilización de la rúbrica, como un instrumento de coevaluación y autoevaluación de este aprendizaje, para que los estudiantes aprendan a construir aquellos conceptos y destrezas necesarias que mejoren su fluidez lectora.

- El desarrollo de las habilidades metacognitivas del lector, mejorando su motivación y capacidad de reflexión sobre la tarea, ayudándole a autorregular aquellos procesos de pensamiento que ponen en juego para desarrollar su fluidez lectora, tales como el conocimiento de las metas que se plantean, o las acciones o estrategias que se utilizan para leer con fluidez.

Tres son las características que definen este enfoque de la evaluación:

a). Enseñar cómo leer con fluidez conlleva que su aprendizaje y la evaluación consiguiente tengan un carácter interactivo, y que la información que se obtiene de esa evaluación retroalimente tanto al alumno como al docente para mejorar el proceso de enseñanza-aprendizaje. En este contexto, por evaluación entendemos todas aquellas actividades que lleva a cabo el docente o el alumno, que proporcionen retroalimentación a ambos, necesaria para implementar la enseñanza (docente) y el 
aprendizaje (alumno) de la lectura fluida. Por ejemplo, cuando el maestro lleva a cabo la actividad de modelar frente a sus alumnos distintos estilos, más o menos fluidos, de lectura (Calero 2013b), para que reflexionen y acuerden cuál es el mejor, les está mostrando y facilitando información pertinente para que ellos la utilicen y traten de acomodar, ajustar y mejorar sus particulares modos de leer fluidamente. En otras palabras, la información que los docentes solemos obtener de actividades de este tipo no solamente sirven para plantear cambios en la enseñanza, sino que también debe ser usada por los alumnos para que ellos puedan tomar decisiones en la mejora de su propio aprendizaje.

b). Un segundo rasgo de la evaluación formativa que aquí planteamos supone implicar al lector en una evaluación compartida con un compañero o con su profesor, y en su propia autoevaluación. El objetivo es que los alumnos cooperen y construyan conjuntamente comprensión sobre cuál es la conducta lectora fluida esperable por sus profesores para mejorar su aprendizaje. En consecuencia, las actividades de coevaluación y autoevaluación mediante rúbricas, orientadas al aprendizaje y al desarrollo de la sensación de autoeficacia, favorecen la creación de dicha comprensión.

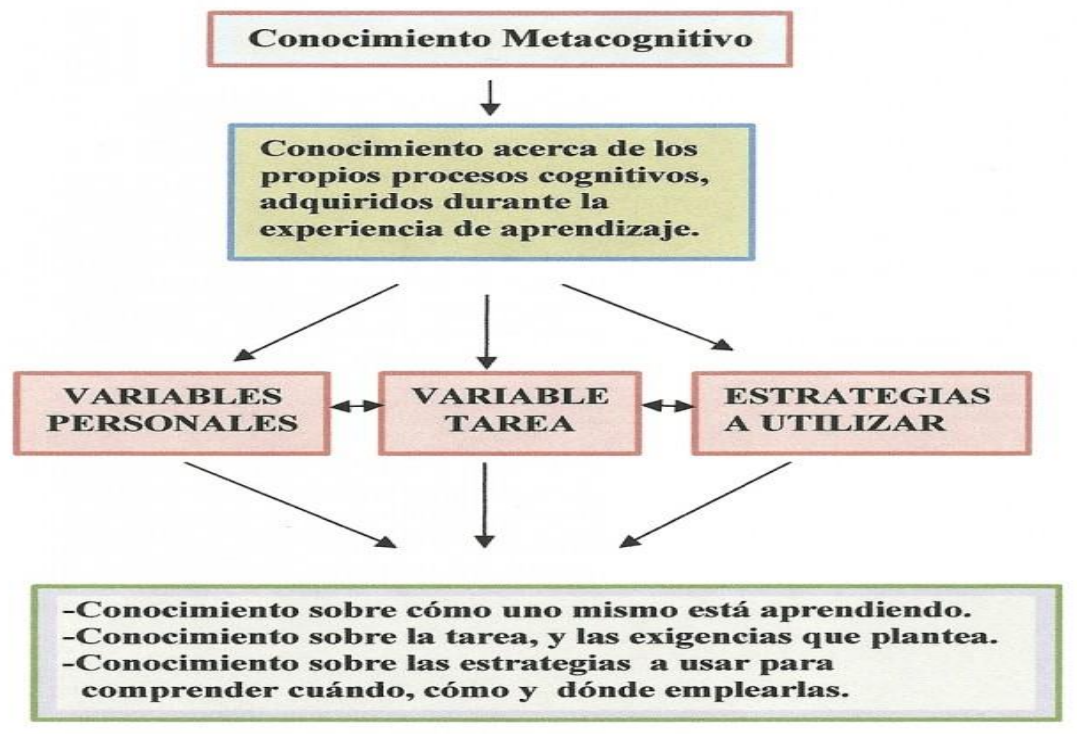

Figura 2: Conocimiento metacognitivo que elaboran los lectores

c). Finalmente, una tercera característica de esta evaluación formativa de la fluidez lectora es que posibilita el desarrollo de conocimiento metacognitivo en el estudiante, para aprender a autorregular ese aprendizaje (figura 2). Dicho

conocimiento se genera sobre tres variables de la lectura fluida: sobre él mismo como lector con un cierto nivel de fluidez lectora; sobre la propia tarea y las exigencias que le plantea (marcas textuales a tener en cuenta, búsqueda de la comprensión); y finalmente sobre las estrategias a utilizar para leer con fluidez (hacer predicciones sobre el texto, ritmo lector, lectura prosódica y resumen del mismo). 


\section{La rúbrica como un instrumento de evaluación formativa de la fluidez lectora. Características}

Dado que la medida de la velocidad al leer no representa una evaluación global, auténtica, cualitativa y formativa de lo que significa una lectura fluida, un instrumento que la investigación actual plantea como idóneo es la rúbrica (o matriz de evaluación). Se trata de una alternativa coherente de evaluación de la fluidez lectora (Simon, 2001), un recurso de evaluación cualitativa y formativa que se constituye actualmente en un instrumento imprescindible para ayudar a diagnosticar e intervenir en la mejora de los procesos de enseñanza-aprendizaje, que puede ser una guía en la evaluación y asignación al estudiante de un nivel de competencia adecuado. Construida con descriptores cualitativos sobre criterios de velocidad lectora, fraseo, pausas, entonación y acento, entre otros, este instrumento trata de establecer la naturaleza del desempeño concreto de un lector en lectura fluida. Por otra parte, proporciona al docente una medida precisa del logro que los lectores alcanzan, en términos del producto final y del proceso que siguen para alcanzar un adecuado nivel de fluidez lectora. Para estos últimos supone una guía explícita y concreta de qué destrezas concretas se espera que adquieran para leer con fluidez. Además, favorece el que se impliquen en este aprendizaje y adquieran, en función de la edad y nivel educativo, una cierta competencia en el seguimiento y control metacognitivo sobre la propia actividad de leer con fluidez, para poder así construir la comprensión del texto que lee. Ese seguimiento y control, asociado a la construcción de la comprensión del texto, se caracteriza por la habilidad que los lectores desarrollan para saber cuándo hacer una pausa entre las distintas frases que leen, cuándo poner énfasis en la lectura de determinadas palabras, o también cuándo elevar o bajar el tono de voz en la lectura del texto en función de los signos de puntuación.

Decimos que la rúbrica se sitúa en un modelo de evaluación formativa, porque ofrece retroalimentación al docente para implementar el proceso de enseñanza, y al propio alumno acerca de su progreso académico. Al implicar a los lectores, les otorga la responsabilidad de autocontrolar y regular el proceso que siguen para mejorar su fluidez lectora, impulsándoles a hacerse cada vez más conscientes de sus puntos débiles y fuertes, a través del manejo de procesos de pensamiento que la propia actividad de la rúbrica les demanda para enseñarles a autoevaluarse.

La rúbrica, se utiliza como un recurso de coevaluación entre alumnos, o entre alumno/profesor, $y$ comprende varios descriptores ( ver figuras 3, 4 y 5), que explican diferentes componentes de la fluidez lectora a evaluar, tales como la lectura expresiva, el tono, el fraseo adecuado, el ritmo lector y la comprensión.

Es importante familiarizar a los estudiantes con el lenguaje que se utilice para explicarles el sentido de los descriptores de una rúbrica, porque son ellos quienes van a utilizarlos para valorar la fluidez lectora de sus compañeros y, por ende, la suya propia. Evidentemente, el uso de una rúbrica exige por parte de quien la utilice (el docente, un alumno, o un familiar) el conocimiento del lenguaje de los descriptores que la definen, para que éste llegue a formar parte del vocabulario que se usa en clase en el desarrollo de la fluidez lectora, y las estrategias a utilizar durante su práctica diaria. De ese modo, los alumnos aprenderán a utilizar ese instrumento como un medio idóneo de desarrollo de la conciencia de autoaprendizaje y autorregulación de su propia capacidad para leer fluidamente.

Los descriptores se gradúan, en función de los diferentes niveles de logro del lector, expresados en términos de una escala (por ejemplo: Excelente, Bueno, Necesita mejorar), o 
alternativamente términos numéricos $(4,3,2,1)$, que al final se pueden sumar para poder determinar una resultado global más concreto en fluidez: (A, B, C; ó nivel Alto, Medio o Bajo).

\subsection{Fases para enseñar a los alumnos a usar las rúbricas}

El uso de la rúbrica conlleva un proceso de andamiaje para transferir al lector la responsabilidad progresiva de su uso, dirigido por el profesor, y durante el tiempo que sea necesario hasta lograr que los lectores se familiaricen con este instrumento de coevaluación. Tres son las fases a seguir:

a). Modelado inicial del uso de la misma por parte del profesor. Es aconsejable elegir cada vez un descriptor y sus distintos niveles de logro, para facilitar que el alumnado se familiarice con su significado y evaluación. Para tal fin, el docente llevará a cabo la lectura de un texto elegido previamente, y les mostrará el sentido que tiene dicho descriptor (por ejemplo: "Lee con expresividad", llevando a cabo distintos tipos de lectura expresiva para que los estudiantes las reconozcan y puedan discriminarlas para evaluarlas). Ese mismo descriptor se trabajará con distintos textos. La reflexión en voz alta del docente sobre cómo él mismo lee y evalúa ese descriptor, es esencial para el objetivo antes señalado de la familiarización del estudiante con su significado y evaluación.

b). Práctica supervisada por parejas. Tras la elección de un texto corto y adecuado al nivel del alumnado, uno de los dos estudiantes lo lee. Al final, el otro consulta la rúbrica y le asigna a su compañero una puntación que defina, según él, el nivel de logro conseguido en cada descriptor. Posteriormente, se cambiarán los papeles y el primer alumno será quien ahora haga de evaluador de su otro compañero. c). Puesta en común con todo el grupo, con el objetivo de que los estudiantes reflexionen sobre los problemas que se hayan encontrado, a la hora de interpretar o utilizar los descriptores o los distintos niveles de logro de la rúbrica que se utilice.

Durante el manejo de una rúbrica, el estudiante puede leer un texto elegido previamente, e incluso grabarlo para ser evaluado. El tiempo de escucha de una lectura no debe sobrepasar los 2 ó 3 minutos. Al acabar la audición, el docente, o el alumno que hace de observador-evaluador pide al estudiante que ha leído que resuma la lectura, consulta la rúbrica, y asigna la puntuación adecuada que se corresponda con el específico tipo de nivel de desempeño que refleja la rúbrica en cada descriptor. Por último, se intercambian los papeles y quien hizo de lector ahora hará de evaluador. Es importante que, quien haya escuchado la lectura, proporcione retroalimentación al estudiante, señalando de cada descriptor, según su criterio, lo que caracteriza la lectura que haya hecho. Estas observaciones ayudarán, tanto al lector como al compañero que está evaluando, a mejorar el control sobre la tarea y sobre sus propios desempeños en la misma.

La rúbrica es sencilla y funcional, no genera mucho gasto de tiempo escolar para el docente, es fácil de utilizar, y su uso frecuente incrementa el control y la regulación del lector de su propio proceso de aprendizaje de lectura fluida. Se aconseja que forme parte del portfolio individual de lectura de cada alumno, como una muestra más de su competencia lectora ante su profesor y sus padres. Quienes tenemos experiencia en el uso de rúbricas para desarrollar la fluidez lectora, sabemos que los lectores acostumbrados a utilizarlas, por una 
parte vuelcan sus recursos cognitivos hacia la construcción de la comprensión del texto, y por otra, ponen en juego otros recursos de tipo metacognitivo que les ayudan a tomar el control, la supervisión y el seguimiento de su competencia en lectura fluida, a partir de los descriptores que se incluyan en las rúbricas. Es sorprendente comprobar cómo estos estudiantes se caracterizan por la habilidad de saber cuándo hacer una pausa entre las distintas frases que leen, cuándo ralentizar el ritmo de lectura para comprender mejor, cuándo poner énfasis en la lectura de determinadas palabras, o cuándo elevar o bajar el tono de voz en la lectura de algunas partes del texto en función de los signos de puntuación, etc,

\subsection{Ejemplos de rúbricas y recursos online para construirlas}

A continuación, presentamos 3 ejemplos de rúbricas, y recomendamos un recurso online que facilita al docente la posibilidad de construirlas y diseñarlas para distintas asignaturas.

En el primer ejemplo, la rúbrica ha sido elaborada, y autorizada su publicación en este artículo, por Víctor Solis, un docente de "The Magellan International School" en Austin, Texas, un centro que ofrece un programa de inmersión en español. Esta rúbrica puede utilizarse con alumnado de los primeros cursos de Educación Primaria, y abarca cuatro descriptores: expresión, ritmo de lectura, precisión en el reconocimiento de palabras y comprensión del texto. Destacar, que los niveles de logro se representan con cuatro dibujos que ejemplifican el proceso de nacimiento de un pollito, lo que podría representar el desarrollo de una destreza cognitiva y metacognitiva, tan importante como es la fluidez lectora.

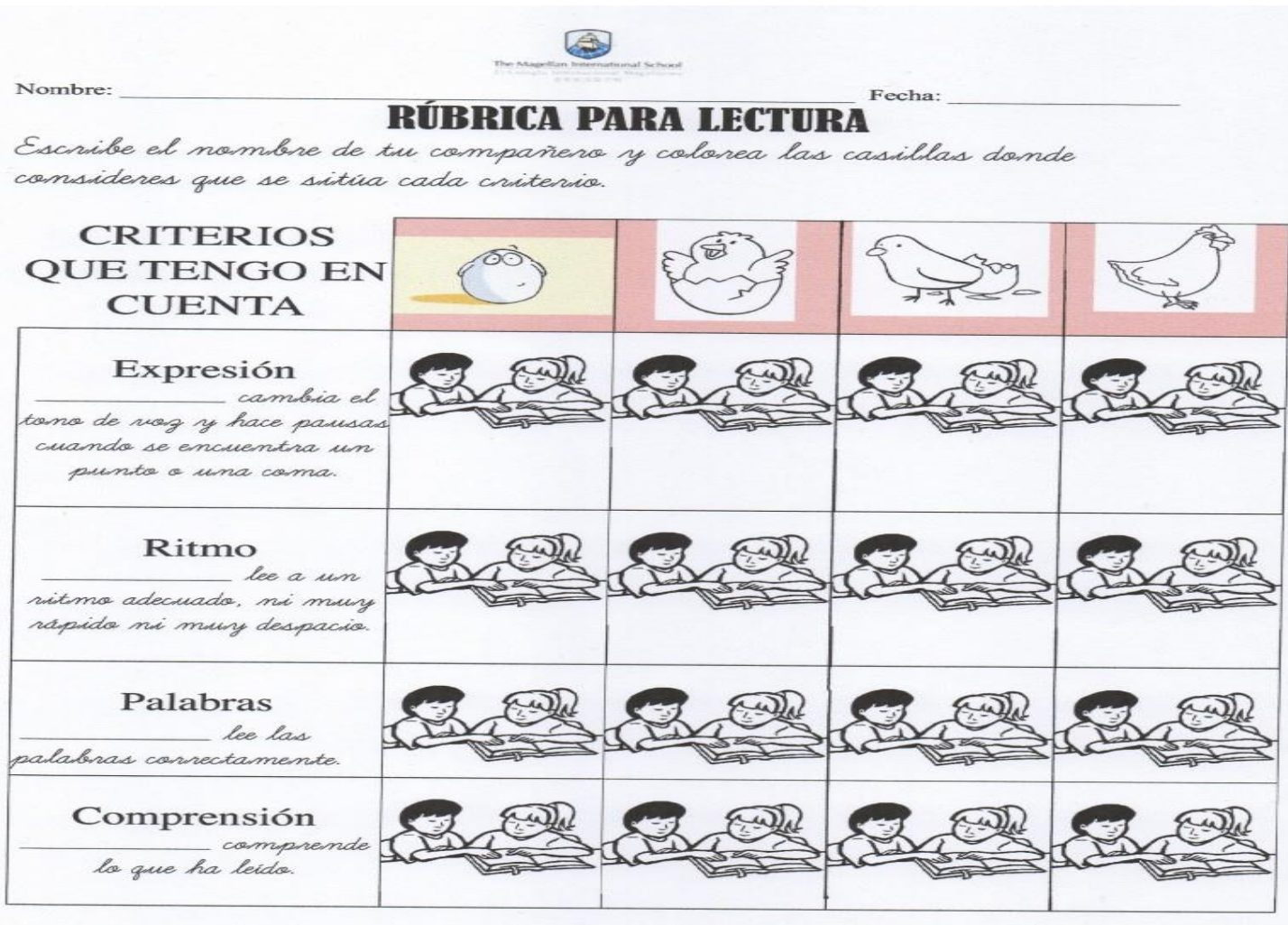


Figura 3. Rúbrica de evaluación del aprendizaje temprano de la fluidez lectora (CVíctor Solís. The Magellan International School, www.comprension-lectora.org .

El segundo ejemplo lo hemos utilizado con alumnado de $2^{\circ}$ de Educación Primaria, y los criterios que la rúbrica recoge están referidos a la expresividad, ritmo lector, pausas y comprensión del texto.

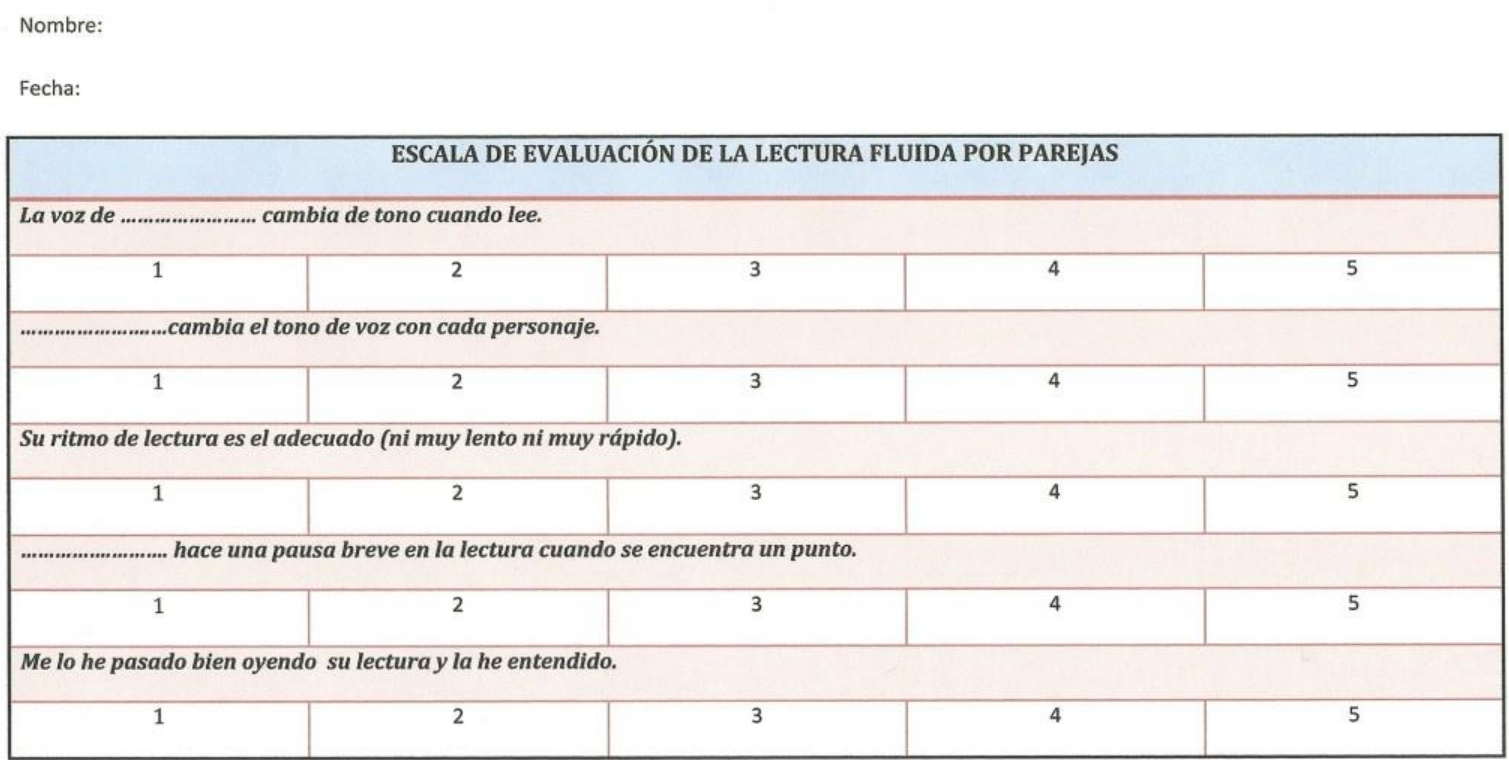

Figura 4. Rúbrica de evaluación de aprendizaje temprano de la fluidez lector (Cursos iniciales de Educación Primaria ) (C) www.comprension-lectora.org

El tercer ejemplo de rúbrica consta de cinco descriptores, con una escala de valoración de logro con cuatro alternativas. Puede usarse con alumnado de cursos intermedios y superiores de Educación Primaria, y ofrece la posibilidad de cuantificar la puntuación de cada alumno, y la del grupo-clase. 
Alumno: Fecha:

Observador: Texto a leer:

\begin{tabular}{|c|c|c|c|c|c|}
\hline & Ritmo de lectura/fraseo. & $\begin{array}{l}\text { Expresividad y } \\
\text { entonación. }\end{array}$ & Pausas al leer. & $\begin{array}{c}\text { Lectura acentuada de } \\
\text { determinadas } \\
\text { palabras del texto. }\end{array}$ & Seguridad al leer. \\
\hline 4 & $\begin{array}{l}\text {-El estudiante lee todo el texto } \\
\text { con ritmo y continuadamente, } \\
\text { prestando atención a todos los } \\
\text { signos de puntuación }(., \ldots \ldots-), y \\
\text { dividiendo el texto en frases con } \\
\text { sentido. }\end{array}$ & $\begin{array}{l}\text {-El estudiante lee todo el } \\
\text { texto con un adecuado } \\
\text { cambio de entonación y } \\
\text { expresividad, para } \\
\text { comprender lo que está } \\
\text { leyendo. }\end{array}$ & $\begin{array}{l}\text {-El estudiante lee todo el } \\
\text { texto haciendo las } \\
\text { correspondientes pausas } \\
\text { para acabar frases, o } \\
\text { atender a los signos de } \\
\text { interrogación y admiración. }\end{array}$ & $\begin{array}{l}\text {-El estudiante lee todo el texto } \\
\text { acentuando la lectura de } \\
\text { aquellas palabras que le } \\
\text { aportan significado. }\end{array}$ & $\begin{array}{l}\text {-El estudiante lee todo el texto } \\
\text { relajado y confiado en su nivel } \\
\text { de lectura, y corrige } \\
\text { fácilmente cualquier error } \\
\text { que comete. }\end{array}$ \\
\hline 3 & $\begin{array}{l}\text {-El estudiante lee la mayor parte } \\
\text { del texto con ritmo, prestando } \\
\text { atención a los signos de } \\
\text { puntuación }(.,-\ldots .)-\text { - }\end{array}$ & $\begin{array}{l}\text {-El estudiante lee la mayor } \\
\text { parte del texto cambiando } \\
\text { adecuadamente la voz y la } \\
\text { entonación para buscar el } \\
\text { significado. }\end{array}$ & $\begin{array}{l}\text {-El estudiante lee la mayor } \\
\text { parte del texto haciendo las } \\
\text { pausas que corresponden a } \\
\text { los signos de puntuación, } \\
\text { interrogaciones o } \\
\text { admiraciones. }\end{array}$ & $\begin{array}{l}\text {-El estudiante lee la mayor } \\
\text { parte del texto resaltando el } \\
\text { acento de algunas palabras } \\
\text { que aportan significado al } \\
\text { mismo. }\end{array}$ & $\begin{array}{l}\text {-El estudiante lee la mayor } \\
\text { parte del texto de un modo } \\
\text { relajado y confiado, y alguna } \\
\text { vez se muestra confundido } \\
\text { con sus errores. }\end{array}$ \\
\hline 2 & $\begin{array}{l}\text {-La lectura del estudiante es unas } \\
\text { veces rápida, y otra con pausas } \\
\text { inesperadas al leer. }\end{array}$ & $\begin{array}{l}\text {-El estudiante lee el texto } \\
\text { con cambios en el tono y la } \\
\text { expresividad, que no se } \\
\text { ajustan al significado del } \\
\text { texto. }\end{array}$ & $\begin{array}{l}\text {-El estudiante hace, en } \\
\text { pocas ocasiones, las } \\
\text { adecuadas pausas cuando se } \\
\text { encuentra signos de } \\
\text { puntuación, interrogaciones } \\
\text { o admiraciones. }\end{array}$ & $\begin{array}{l}\text {-El estudiante, en pocas } \\
\text { ocasiones, resalta el acento de } \\
\text { algunas palabras del texto. }\end{array}$ & $\begin{array}{l}\text {-El estudiante se muestra a } \\
\text { veces nervioso y confundido } \\
\text { con sus errores. }\end{array}$ \\
\hline 1 & $\begin{array}{l}\text {-El estudiante lee con grandes } \\
\text { pausas, o leyendo lentamente las } \\
\text { palabras del texto. }\end{array}$ & $\begin{array}{l}\text {-El estudiante lee el } \\
\text { texto de un modo } \\
\text { monótono, sin entonación } \\
\text { y expresividad. }\end{array}$ & $\begin{array}{l}\text {-El estudiante no hace pausa } \\
\text { alguna cuando se encuentra } \\
\text { con signos de puntuación, } \\
\text { interrogaciones o } \\
\text { admiraciones. }\end{array}$ & $\begin{array}{l}\text {-El estudiante no atiende a } \\
\text { ninguna palabra del texto } \\
\text { para resaltar su lectura. }\end{array}$ & $\begin{array}{l}\text {-El estudiante se muestra } \\
\text { nervioso al leer. }\end{array}$ \\
\hline 를 를 & & & & & \\
\hline
\end{tabular}

Figura 5. Rúbrica de evaluación del logro en fluidez lectora (Cursos intermedios y superiores de Educación Primaria) (C) www.comprension-lectora.org

Finalmente, Rubistar es un recurso online gratuito para los docentes, que puede ayudarles a crear y diseñar rúbricas personalizadas, no sólo para el ámbito del desarrollo de la lectura, sino también para otras áreas del aprendizaje.

\section{Conclusiones}

Aunque existen suficientes evidencias experimentales que resaltan la importancia de la necesidad del desarrollo temprano de la fluidez lectora, como una destreza básica para que los alumnos de Educación Primaria adquieran una competencia adecuada en comprensión lectora, en muchas aulas aún se sigue asociando velocidad con fluidez lectora. Esa asociación ha condicionado su instrucción, y los procedimientos de evaluación que se utilizan en las aulas para medir el logro en ese aprendizaje.

En este trabajo hemos propuesto un enfoque del desarrollo de la fluidez lectora, que tiene en cuenta las capacidades cognitivas y metacognitivas del alumnado, que considera la lectura fluida como una destreza de vínculo entre las habilidades de decodificación del niño y la comprensión lectora, y que se define como un proceso simultáneo y autorregulado de aprendizaje del automatismo en el reconocimiento de palabras, la lectura prosódica y la comprensión del texto. 
Concluimos que la utilización de procedimientos auténticos y significativos de enseñanza de la lectura fluida, junto con el uso de la rúbrica como un procedimiento de evaluación formativa, ya desde los primeros cursos de esa etapa educativa, son una garantía para que los lectores logren un buen ritmo lector, una lectura prosódica, y una mejora de sus resultados en lectura comprensiva. 


\section{Bibliografía}

- Calero, A. (2012). Cómo mejorar la comprensión lectora. Estrategias para lograr lectores competentes. Wolters Kluwer. Madrid.

- Calero, A. (2013a). La lectura repetida: Una estrategia de aprendizaje temprano de la fluidez lectora. En http://comprension- lectora.org/la-lectura-repetida-unaestrategia-de-aprendizaje-temprano-de-la-fluidez-lectora/ y http://comprensionlectora.org/relacion-familia-colegio-y-desarrollo-de-la-fluidez-lectora/ . 14/05/2013

- Calero, A. (2013b) ¿Sabes cómo modelar la lectura fluida de tus alumnos?- En http://comprension-lectora.org/como-modelar-la-lectura-fluida/ . 14/05/2013

- Christophe, A., Dupoux, E., Bertoncini, J. \& Meller, J. (1994). Do infants perceive word boundaries? An empirical study of the bootstrapping of lexical adquisition. En The Journal of the Acoustical Society of America, 95 (3) 1570-1580-

- González Trujillo (2005) Tesis doctoral. Departamento de Psicología Evolutiva. Universidad de Granada. http://hera.ugr.es/tesisugr/15808932.pdf .14/05/2013

- Harris, T.L. \& Hodges, R.E. (eds) (1995). The literacy dictionary: The vocabulary of reading and writing. Newark, DE. International Reading Association.

- Hirsh-Pasek, K., Kemler Nelson, D. G., Jusczyk, P. W., Wright Cassidy, K., Druss, B., \& Kennedy, L. (1987). Clauses are perceptual units for young infants. En Cognition, 26, 269-286.

- Kuhn, M.R., \& Stahl, S. (2003). Fluency: A review of developmental and remedial strategies. En The Journal of Educationai Psychology. 95,1-19

- LaBerge, D., \& Samuels, J. (1974). Towards a theory of automatic information processing in reading. Cognitive Psychology, 6, 293-323.

- del Mazo, A. (2013). ¿Cúantas palabras lee por minuto? En http://queduquequeducuando.blogspot.com.es/2012/12/cuantas-palabras-lee-porminuto.html . Descargado el 14/05/2013 
- Miller, J., Schwanenflugel, P. (2006) Prosody of Syntactically Complex Sentences in the Oral Reading of Young Children. En Journal of Educational Psychology, 98(4), 839-853.

- Pikulski, J.J. \& Chard, D.J. (2005). Fluency: Bridge between decoding and reading comprehension. En The Reading Teacher, (58), 6, 510-519.

- Rasinski, T.V. \& Samuels, J. (2011a). Reading fluency: What it is and what it is not. En What Research Has to Say About Reading Instruction, IV Edition, 94-114. Samuels \& Farstrup (eds). International Reading Association.

- Rasinski, T.V., Reutzel, R., Chard, D., Linan, S. (2011b). Reading fluency. En Handbook of Reading Research, Vol. IV, 286-319.

- Ravid D, Mashraki YE. Prosodic reading, reading comprehension and morphological skills in Hebrew-speaking fourth graders. Journal of Research in Reading. 2007;30(2):140-156

- Rubistar. http://rubistar.4teachers.org/index.php?lang=es

- Samuels, S.J. (2007). The DIBELS tests: Is speed of barking at print what we mean by fluency? En Reading Research Quarterly, 42, 563-566.

- Simon, M. y Forgette-Giroux, R. (2001). A rubric for scoring postsecondary academic skills. En Practical Assessment, Research \& Evaluation, 7 (18). En http://pareonline.net/getvn.asp?v=7\&n=18 . Descargado el 14/05/2013. 